\title{
Pneumotórax recidivante secundário à agenesia do diafragma*
}

\author{
Camila Baracat Mendina ${ }^{1}$, Paulo Manuel PÊGo-Fernandes², Fábio Biscegli Jatene ${ }^{2}$
}

Os autores relatam um caso de pneumotórax recidivante secundário à agenesia do diafragma em mulher de 36 anos de idade. O diagnóstico foi realizado pela história clínica e radiografia de tórax. Optada pela correção cirúrgica, foi diagnosticada a agenesia de diafragma e realizada pleurodese. No segundo mês de pós-operatório, perante um novo episódio de pneumotórax, optou-se por laqueadura das trompas para impedir a entrada de ar no abdome no período ovulatório e, conseqüentemente, evitar novos episódios de pneumotórax. (J Pneumol 2002;28(1):48-50)

\section{Recurrent pneumothorax secondary to agenesis of the diaphragm}

The authors report a case of recurrent pneumothorax secondary to agenesis of the diaphragm in a 36-year old female. The diagnosis was established by means of clinical history and chest X-ray examination. Surgical correction was carried out. Diaphragmatic agenesis was detected during the procedure and pleural abrasion was performed. Two months following surgery another pneumothorax episode occurred. Fallopian tube ligature was then performed in order to prevent the entrance of air into the abdomen during ovulation, and thus avoid further recurrences of pneumothorax.

Descritores - Diafragma. Agenesia. Pneumotórax. Recidiva. Key words - Diaphragm. Agenesis. Pneumothorax. Recurrence.

\section{INTRODUÇÃO}

A agenesia de diafragma constitui afecção rara, levando ao óbito nas primeiras horas de vida extra-uterina, geralmente por insuficiência respiratória, uma vez que o conteúdo abdominal invade o tórax, impedindo o desenvolvimento do pulmão fetal. Por esse motivo, a constatação de agenesia diafragmática em adultos é rara.

O pneumotórax recidivante é mais freqüente em adultos do sexo masculino, longilíneos e fumantes, e a etiologia mais comum são as blebs subpulmonares.

* Trabalho realizado no Serviço de Cirurgia Torácica - Instituto do Coração do Hospital das Clínicas da Faculdade de Medicina da Universidade de São Paulo.

1. Graduanda da Faculdade de Medicina da Universidade de Mogi das Cruzes, SP.

2. Professor Livre-Docente da Faculdade de Medicina da Universidade de São Paulo, SP.

Endereço para correspondência - Prof. Dr. Paulo Manuel Pêgo Fernandes, InCor-Instituto do Coração do HCFMUSP, Divisão de Cirurgia, $2^{\circ}$ andar, Av. Dr. Enéas de Carvalho Aguiar, 44 - 05403-000 - São Paulo, SP, Brasil. Tel./Fax: (11) 3069-5248; e-mail: paulopego@incor. usp.br

Recebido para publicação em 11/6/01. Aprovado, após revisão, em 27/7/01.
Devido à raridade das lesões, apresentamos um caso de agenesia de diafragma e pneumotórax recidivante em uma paciente de 36 anos de idade.

\section{RELATO DO CASO}

Mulher de 36 anos de idade com relato de episódios de pneumotórax recidivantes nos últimos dois anos.

Os episódios eram mensais e coincidentes com o ciclo menstrual. Radiografia e tomografia computadorizada torácicas revelavam pneumotórax à direita com elevação desta cúpula diafragmática (Figuras 1, 2 e 3) e presença de conteúdo herniário (Figura 4). Foi feito o diagnóstico de pneumotórax catamenial e por isso planejada cirurgia de pleurodese com abrasão pleural e talcagem através de pequena toracotomia direita. Entretanto, após a abertura da pleura parietal, foi constatada agenesia do diafragma direito, além de pulmão e fígado discretamente hipoplásicos (Figura 5).

Visando prevenir recidiva de pneumotórax futuro, foi realizada a pleurodese com abrasão pleural e talcagem. A paciente teve boa evolução no pós-operatório imediato. No entanto, no segundo mês pós-operatório, após dois dias de fortes dores no hipocôndrio direito, a paciente apresentou novo pneumotórax (menor que os anteriores) seguido de alívio da dor abdominal.

Como a paciente já possuía os filhos desejados, foi realizada a laqueadura das trompas. Após essa nova cirurgia, a paciente permanece assintomática por oito anos. 


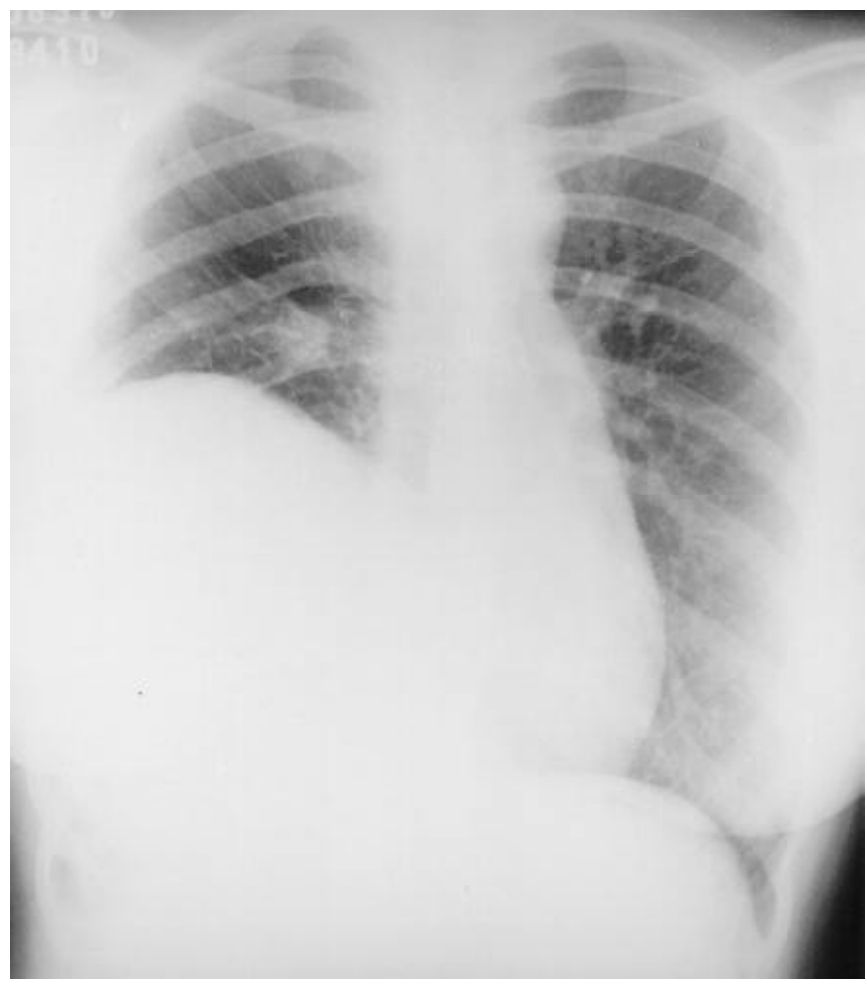

Figura 1 - Radiografia de tórax (póstero-anterior) mostrando a presença de conteúdo herniário

\section{DISCUSSÃO}

A agenesia de diafragma constitui um grupo dentro da classificação das hérnias diafragmáticas congênitas, cuja incidência subiu de uma em 7.000 para uma em 2.000(1).

É uma condição extremamente rara em adultos - com apenas três casos relatados na literatura inglesa ${ }^{(1)}$ - pela alta mortalidade logo após o nascimento, pois está, na maioria das vezes, associada à hipoplasia pulmonar, que causa falência respiratória progressiva até a morte ${ }^{(2)}$. No caso relatado, acreditamos que o fato de o fígado ser hipodesenvolvido facilitou o crescimento pulmonar, possibilitando a vida após o nascimento.

Sugere-se que esse problema constitui uma entidade separada de herança autossômica recessiva com causas diferentes ${ }^{(3)}$. Entretanto, não se sabe exatamente em qual estágio do desenvolvimento intra-uterino o defeito ocor$r e^{(3)}$. Na maioria das vezes, o defeito no diafragma está no lado esquerdo na região dorsolateral, possuindo contorno bem definido, o que geralmente possibilita o repa$\mathrm{ro}^{(2)}$.

Grandes defeitos diafragmáticos foram corrigidos usando-se vários métodos: sutura com lobo hepático, enxertos, "dobra" com músculo abdominal, materiais protéti$\cos ^{(2)}$. Uma malha de polipropileno tende a incorporar-se no crescimento das fibras e é mais indicada para reparar grandes defeitos ${ }^{(2)}$.

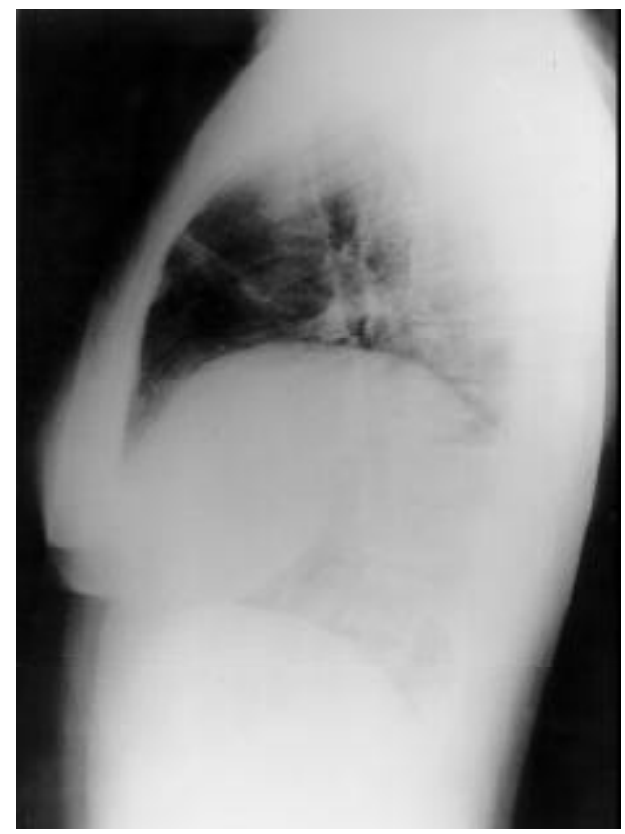

Figura 2

Radiografia de tórax (perfil) mostrando a presença de conteúdo herniário
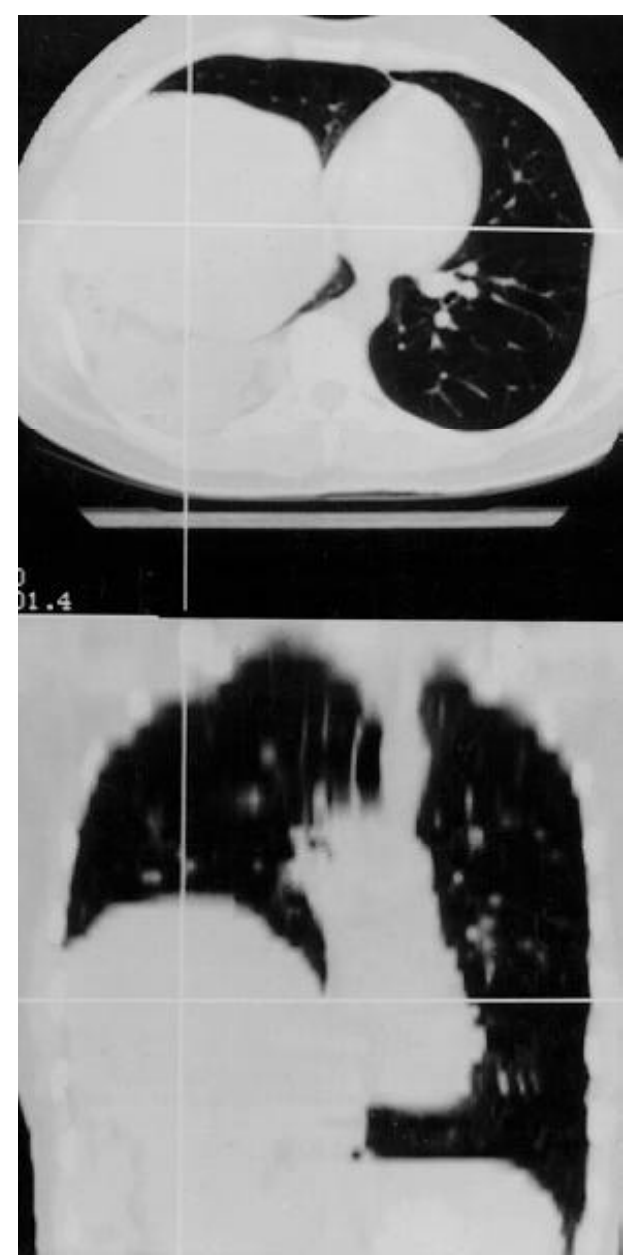

Figura 3

Tomografia computadorizada de tórax mostrando a presença do figado hipoplásico e conteúdo herniário 


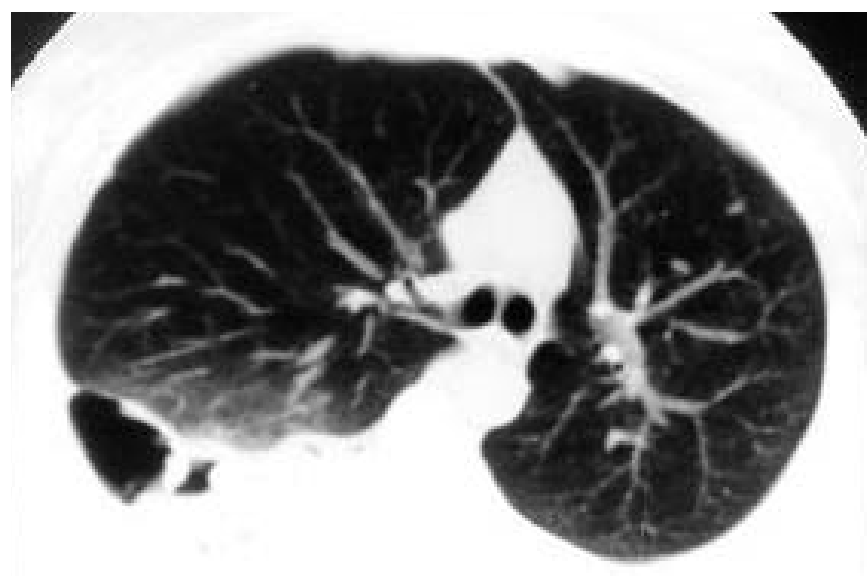

Figura 4 - Tomografia computadorizada de tórax mostrando, ao nível da carina, presença de conteúdo herniário (alças intestinais)

Foi relatado um caso de agenesia de hemidiafragma esquerdo em um homem assintomático. Por esse motivo, nenhum tratamento fora necessário porque o movimento livre dos órgãos intra-abdominais não provocava encarceramento do intestino ${ }^{(4)}$.

Já no caso apresentado - paciente também assintomática - acreditamos que o fígado funcionava como um "pseudodiafragma", não tendo sido necessária correção cirúrgica.

Por outro lado, o traço característico do pneumotórax catamenial é pneumotórax recorrente na quarta década de vida, na maioria das vezes do lado direito e coincidente com o período menstrual(5). Os sintomas mais freqüentes são dor torácica ou dispnéia ou ambos; no entanto, algumas pacientes são assintomáticas ${ }^{(6)}$. Por esse motivo, acreditamos tratar-se de pneumotórax catamenial. O diagnóstico é clínico e radiológico ${ }^{(5)}$.

A causa é desconhecida. Uma hipótese fisiopatológica envolve passagem de ar do trato genital através de brechas no diafragma ${ }^{(5,7)}$. Por isso, acreditamos que, no pósoperatório, a dor abdominal apresentada pela paciente tenha sido um pneumoperitônio, aliviado concomitantemente com a formação de um novo pneumotórax (possível pela agenesia diafragmática).

A afecção está muito associada à endometriose diafragmática, mesmo sendo o diafragma um sítio muito raro. Neste caso, o diagnóstico é sugerido por recorrentes hemoptises durante o ciclo menstrual ${ }^{(5)}$.

O pneumotórax catamenial é manuseado exatamente do mesmo modo que os outros tipos de pneumotórax. Quando a paciente é assintomática, o tratamento é conservador. Por outro lado, se o pneumotórax é maior que $20 \%$ e cursa com sintomas importantes, faz-se necessá-

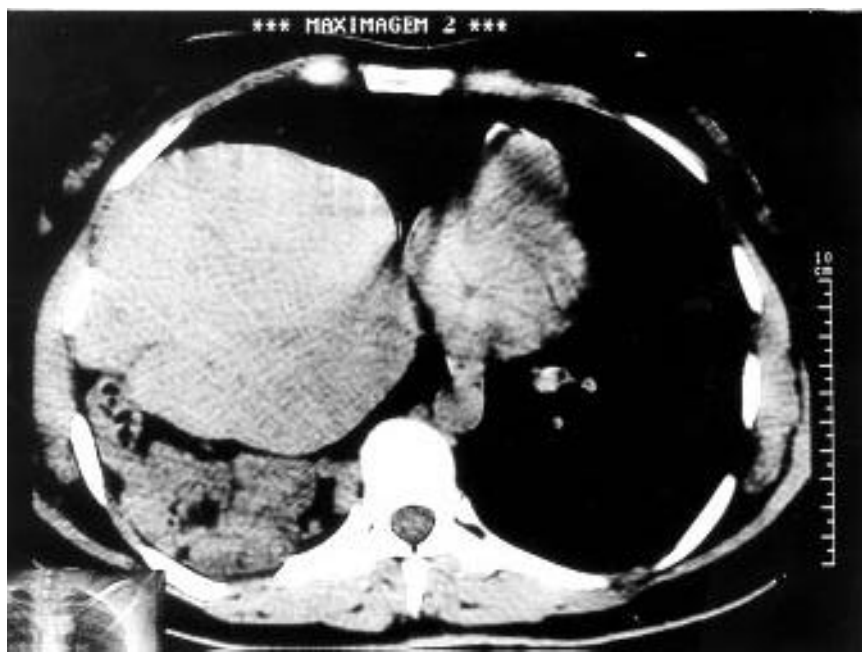

Figura 5 - Tomografia computadorizada de tórax mostrando o figado hipoplásico. Posteriormente ao figado, presença de alças intestinais.

ria a drenagem torácica e analgesia. Uma variedade de opções de tratamento preventivos existe, como contraceptivos orais para suspender a ovulação; entretanto, recaídas podem ocorrer. Ooforectomia bilateral, danazol e, recentemente, análogos da gonadotrofina mostraram melhores resultados no tratamento preventivo(6). Segundo Fonseca, foi notada a persistência de pneumotórax recorrentes em oito pacientes submetidas à histerectomia ${ }^{(8)}$.

Em consenso, é recomendado que, depois da sínfise pleural, um regime terapêutico de análogo de gonadotrofina seja implantado para prevenir novos episódios de pneumotórax, especialmente em pacientes que desejam preservar seu potencial reprodutivo(6). Em nosso caso, como a paciente já possuía os filhos desejados, foi realizada a laqueadura das trompas.

\section{REFERÊNCIAS}

1. Travaline JM, Cordova F. Agenesis of the diaphragm. Am J Med 1996; 100:585

2. Singh G, Bose M. Agenesis of hemidiaphragm in adults. Aust N Z J Surg 1993;63:327-8.

3. Hatzitheofilou C, Conlan AA, Nicolaou N. Agenesis of the diaphragm. S Afr Med J 1982;62:999-1001.

4. Tzelepis MD, Ettensohn DB, Shapiro B, McCool D. Unilateral absence of the diaphragm in an asymptomatic adult. Chest 1988;94:1301-3.

5. Guerin JC. L'endometriose pleuro-pulmonaire. Rev Pneumol Clin 1999; 55:259-61.

6. Blanco S, Hernando F, Gómez A, González MJ, Torres AJ, Balibrea JL. Catamenial pneumothorax caused by diaphragmatic endometriosis. J Thorac Cardiovasc Surg 1998;116:179-80

7. Kirschner PA. Catamenial pneumothorax: a unifying concept. Ann Thorac Surg 2000;69:1644

8. Fonseca P. Catamenial pneumothorax: a multifactorial etiology. J Thorac Cardiovasc Surg 1998;116:872-3. 